\title{
Response modeling of small-scale energy consumers for effective Demand Response Applications
}

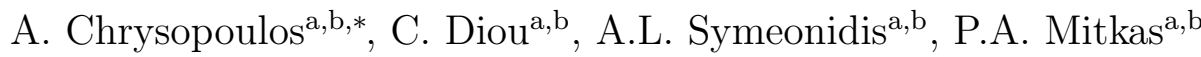 \\ ${ }^{a}$ ECE Department, Aristotle University of Thessalonki, Thessalonki, Greece \\ ${ }^{b}$ Information Technologies Institute, CERTH, Thessalonki, Greece
}

Abstract

The Smart Grid paradigm can be economically and socially sustainable by engaging potential consumers through understanding, trust and clear tangible benefits. Interested consumers may assume a more active role in the energy market by claiming new energy products/services on offer and changing their consumption behavior. To this end, suppliers, aggregators and Distribution System Operators can provide monetary incentives for customer behavioral change through Demand Response programs, which are variable pricing schemes aiming at consumption shifting and/or reduction. However, forecasting the effect of such programs on power demand requires accurate models that can efficiently describe and predict changes in consumer activities as a response to pricing alterations. Current work proposes such a detailed bottom-up response modeling methodology, as a first step towards understanding and formulating consumer response. We build upon previous work on small-scale consumer activity modeling and provide a novel approach

\footnotetext{
${ }^{*}$ Corresponding author. Tel.: +30 2310996349, Fax: +30 2310996398

Email addresses: achryso@issel.ee.auth.gr (A. Chrysopoulos), diou@iti.gr (C. Diou), asymeon@issel.ee.auth.gr (A.L. Symeonidis), mitkas@auth.gr (P.A. Mitkas)
} 
for describing and predicting consumer response at the level of individual activities. The proposed models are used to predict shifting of demand as a result of modified pricing policies and they incorporate consumer preferences and comfort through sensitivity factors. Experiments indicate the effectiveness of the proposed method on real-life data collected from two different pilot sites: 32 apartments of a multi-residential building in Sweden, as well as 11 shops in a large commercial center in Italy.

Keywords: Smart Grid, Small-Scale Consumer Models, Response Modeling, Response Simulation, Demand Response Applications

\section{Introduction}

Due to the gradual restructuring and deregulation of Energy Markets, all the participating entities (utilities, energy suppliers, Distribution System Operators (DSOs) etc.) have to attune their philosophy and their operation model to the changing market landscape. In addition, the new upcoming Smart Grid paradigm necessitates a perfect balance between supply and demand in real time [1].

Even so, the economical and social sustainability of Smart Grids also requires the active involvement of electricity consumers: they have to recognize the added value of new market system technologies and be willing to change their consumption behavior when needed. For example, consumers could reduce or shift their demand over time in response to electricity prices. Additionally, they can choose among a wider range of providers (energy retailers, aggregators etc.) and power options (e.g. green electricity and power quality premiums). 
In the past, energy stakeholders have made little effort to attract the interest of small-scale consumers, even though the residential sector accounts for a significant segment of the overall energy consumption worldwide [2]. Thus, all consumers under contract with a certain energy supplier or aggregator are provided with the same prices, services and communication policies, without taking into account potential groups of consumers with similar needs or behavior. For example, a consumer may not be able to shift his/her consumption according to price signals and benefit from Demand Response (DR), if the proposed program isn't taking into consideration his/her consumption behavioral patterns.

Modeling and shaping demand assumes the ability to predict small-scale consumer response due to pricing policy changes. This is a complex task, since it involves several factors such as consumers' comfort, environmental awareness and sensitivity to monetary incentives. Nevertheless, the gains of successfully modeling response behavior in an activity- and comfort-based context would be substantial, since small changes in individual consumers' behavior can have a major impact on the aggregate power demand. Furthermore, correct consumer segmentation based on these attributes contribute to an open and competitive retail market, as it implies: (i) more tailored electricity services to meet consumers' needs, with a higher rate of acceptance of new products and services, and (ii) the possibility to target energy-savvy consumers as early adopters of new technologies.

Lately, consumer response behavior has been heavily studied and analyzed, in an attempt to better understand and predict the impact of various DR programs to energy markets and power systems in general. To this end, 
different approaches of response modeling were presented recently ([3, 4, 5, 6, 7, 8, 9, 10]). The research that is most closely related to the context of this work was presented in [11]. Yusta et al. proposed a simple mathematical response model to calculate the optimal pricing rates for electricity, assuming "default" customers under different scenarios. Five different mathematical functions for the consumer response were defined: linear, hyperbolic, potential, logarithmic and exponential. Their target was to simulate the hourly changes in consumer response according to the load level, the price of electricity, and the elasticity at every hour.

To this direction, our paper introduces an innovative response modeling approach for small-scale consumers, based on previous work done by the authors on activity modeling [12]. Appliance-level consumption measurements are used to build detailed stochastic activity and appliance models that aggregate to small-scale consumer models. Next, various response models are formulated and applied on the extracted activity models in order to discover useful patterns or information for the consumer behavior (at activity level), taking into consideration consumer comfort and monetary sensitivity. The implemented response models may correspond to predefined, parametric response patterns, or may be computed directly from consumption measurements.

It should be mentioned that the proposed method was extensively evaluated on real measurement data collected from two DR pilot cases implemented in the context of CASSANDRA project 1 , one in a multi-residential building and a second one in a large commercial center. Results indicate that the proposed modeling approach: (i) enables the implementation of a large variety

\footnotetext{
${ }^{1}$ http://cassandra-fp7.eu
} 
of response types without introducing too much complexity, and (ii) leads to accurate response prediction at installation level.

The main contributions of the presented work therefore are:

1. The introduction of a set of data-driven response models that can be used to evaluate the reaction of consumers to pricing signals. These models are built on the basis of results of several DR pilot cases [13], that show how consumption patterns change by altering pricing policies and providing feedback. These models rely solely on electricity measurements, contrary to most state of the art response modeling approaches, that use statistics / demographic data or require additional input.

2. The introduced response models are detailed enough to estimate response behavior for each individual consumption activity. This enables the application of targeted (personalized) pricing schemes, aiming at specific peak shifting or energy reduction targets.

3. The evaluation of the proposed models in two real-world pilot cases: one consisting of apartments in a multi-residential building and a second one consisting of businesses in a large commercial center (mall).

The rest of the paper is organized as follows. Section 2 introduces our approach for response modeling, including the theoretical background and the proposed methodology. In Section 3, a set of ad-hoc response models based on the proposed theoretical formulation, as well as a set of models based on response data measurements are defined and implemented. Section 4 presents the experimental evaluation of the proposed approach, while Section 
5 summarizes work performed, probes on directions for further research, and concludes the paper.

\section{Consumer Response Modeling}

Response modeling aims to indicate whether a consumer will respond to a specific incentive or not, as well as how. As discussed in authors' previous work [12], consumer consumption behavior can be described by a set of consumer activity models

$$
\mathcal{A}_{a}=\left\{\mathbf{a}, \mathbf{f}_{N}(n), \mathbf{f}_{s}(t), \mathbf{f}_{d}(t)\right\}
$$

where $a$ is an activity that may involve a set of one or more appliances a, the start time probability distribution $\mathbf{f}_{s}(t)$ (assuming the activity takes place), the probability distribution of the activity duration, $\mathbf{f}_{d}$, and the probability distribution of the number of times the activity takes place during a day, $f_{N}(n)$. In [12] a detailed computational approach was presented that leads to the estimation of the distributions $\mathbf{f}_{s}, \mathbf{f}_{d}$ and $\mathbf{f}_{N}$ from measurement data (i.e. training of the activity models), along with an estimate of the expected power consumption $P_{a}(t)$ of the activity during the day.

In this work, we study ways to modify the consumer activity models in order to simulate consumer response to pricing incentives. Thus, the objective of the proposed models can be summarized as follows:

Objective: Given a set of activity models trained using data collected under certain (baseline) pricing conditions, our goal is to estimate the set of updated activity models that best describes the consumer 
activities, when pricing conditions change.

The following subsection first provides an overview of the key factors affecting consumer response, as they were identified through analysis of previous research projects and DR pilots [13], while later subsections formulate these concepts into an applied response modeling methodology.

\subsection{Factors Affecting Consumer Response}

Consumer response depends on changes in energy prices, however it also strongly depends on the sensitivity of the consumer to monetary incentives.

\subsubsection{Pricing}

Money is probably the most significant factor for behavioral change in electrical energy consumption. For response modeling, we assume a pricing scheme price $(t)$ that varies depending on the time of day $t$. Alterations in behavior are motivated by changing the baseline pricing scheme price $_{\text {base }}(t)$ (i.e. the existing pricing scheme used when modeling the base load, which is commonly a fixed cost pricing policy) to a new pricing scheme price $_{n e w}(t)$. In our case, consumer response is estimated with the help of two (pricing) ratios: price ratio and daily energy cost ratio.

Price ratio $r(t)$ is used to control activity shifting, namely the start time of the activities as a response to the price change. It is defined as the ratio of the new (DR) and the baseline pricing scheme for each minute of the day:

$$
r(t)=\frac{\text { price }_{\text {new }}(t)}{\text { price }_{\text {base }}(t)}
$$

This metric captures the fluctuations in energy pricing at each point in time. An example is given in Figure 1. 
The calculation of the daily energy cost is achieved via the energy ratio $E_{r}(a)$. This ratio is defined as the daily cost of energy consumed by an activity $a$ with the new pricing scheme divided by the daily cost of energy consumed by the same activity with the baseline pricing scheme:

$$
E_{r}(a)=\frac{\sum_{t=1}^{1440} P_{a}(t) \cdot \text { price }_{\text {new }}(t)}{\sum_{t=1}^{1440} P_{a}(t) \cdot \text { price }_{\text {base }}(t)}
$$

where $P_{a}(t)$ is the average (expected) power consumption of activity $a$ at time $t$. The energy ratio affects the number of times an activity is executed during a day. Notice that contrary to $r(t), E_{r}(a)$ depends on the activity $a$. This is because activities are performed at different times throughout the day (i.e., have different $P_{a}(t)$ ), and therefore a variable pricing scheme affects each activity differently.

\subsubsection{Sensitivity Factor}

In order for a change in behavior to occur, the consumers must be aware, but also sensitive towards price modifications. The sensitivity factor is a set of real values in the $[0,1]$ range that provides an indication on a consumer's interest in money or comfort (for each activity and for a certain period of the day). This factor affects consumer behavior change probability for an activity $a$ after a change in price and/or available information has occurred. In our approach, we define two different types of sensitivity factors: sensitivity to time shifting $s_{t}(a, t)$ and sensitivity to energy reduction $s_{e}(a)$.

Sensitivity to time shifting $s_{t}(a, t)$ indicates the consumers' shifting capability from time period $t$ to another period of the day for activity $a$, in case it is suggested by the new pricing scheme. This action may lead to 
cost reduction, but it may also affect the consumer convenience. On the other hand, sensitivity to energy reduction $s_{e}(a)$ describes the consumers' willingness to increase or reduce the overall daily consumption, by changing the number of times performing an activity or using an appliance. It should be noted that we are assuming independence between the two sensitivity factors, since the are affecting different aspects of the consumers' behavior.

The following subsections pieces together activity models, price modifications and consumer sensitivity into the proposed consumer response modeling methodology focused on time shifting and daily activity frequency response modeling.

\subsection{Time Shifting Response Modeling}

Time shifting models describe the changes in $\mathbf{f}_{s}(t)$ as a result of changing the applied pricing scheme. The degree of change is assumed to depend upon the price ratio $r(t)$ (Equation 2) and consumer's sensitivity to time shifting $s_{t}(a, t)$ described in previous subsections. Also, consumers have specific time shifting preferences for their activities, categorized in the following types:

1. Proximity preference: Consumers prefer to carry out their activities as close to the previously preferred time as possible (while at the same time avoiding the increased cost). Usually, they perform their activities right before or after a price increase.

2. Time frames preference: Consumers prefer to carry out an activity during specific time periods of the day. If they choose to shift their activities as a result of a change in pricing, they perform their activities during a set of preferred time frames of the day. 
According to the proposed model, proximity preference is modeled by a value $s_{t}(a, t)$ that is high around the increased price window $W$, while it is lower (or zero) during the rest of the day. Similarly, time frames preference is modeled by providing high sensitivity values at the preferred day periods and zero sensitivity values the rest of the day.

Thus, the updated start time distribution for activity $a$ after applying a new pricing scheme is given by:

$$
\mathbf{f}_{s_{a}}^{\prime}(t)=\mathbf{f}_{s_{a}}(t) \cdot\left(1+\operatorname{shift}\left(s_{t}(a, t), r(t)\right)\right)
$$

The shifting function shift $\left(s_{t}(a, t), r(t)\right)$ models the dependence of the consumer behavioral change to the different factors of response behavior (time shifting sensitivity and price ratio) given some limitations: (i) there must be limits to the shifting capabilities, namely there should be a lower and an upper threshold that won't allow unrealistic shifting, and (ii) in case no change in pricing has occurred, no load shifting should take place.

There are several mathematical functions that could be used as shifting functions, for example sigmoid functions, piecewise linear functions, parabolic functions etc. In this work, a piecewise linear function was selected as our shifting function, since (i) it can be easily parametrized and (ii) it provides the flexibility needed for the models (e.g. different level of sensitivity for penalties and rewards, thresholds for large values etc). In addition, the piecewise linear function performed well in the response modeling experiments for both datasets examined in this paper. 
Generally, these functions are defined by equation:

$$
\operatorname{shift}\left(s_{t}(a, t), r(t)\right)= \begin{cases}c_{2} \cdot s_{t}(a, t) & r(t)<c_{1} \\ \frac{c_{2}}{c_{1}-1} \cdot(r(t)-1) \cdot s_{t}(a, t) & c_{1}<=r(t)<1 \\ \frac{c_{4}}{c_{3}-1} \cdot(r(t)-1) \cdot s_{t}(a, t) & 1<=r(t)<c_{3} \\ c_{4} \cdot s_{t}(a, t) & r(t)>c_{3}\end{cases}
$$

where $c_{1}, c_{3} \neq 1$. Due to unavailability of detailed response data, we preselected certain values for the four constant parameters of the piecewise function which turned out to fit well for the problem at hand. Figure 2 illustrates the piecewise linear function selected for our modeling approach.

After estimating the resulting values for each minute of the day, $\mathbf{f}_{s}^{\prime}(t)$ is normalized 2 :

$$
\hat{\mathbf{f}} s_{a}(t)=\frac{\mathbf{f}_{s_{a}}^{\prime}(t)}{\sum_{t=1}^{1440} \mathbf{f}_{s_{a}}^{\prime}(t)}
$$

\subsection{Daily Activity Frequency Response Modeling}

In addition to activity time shifting, consumers may respond by reducing or increasing the number of times they perform some of their activities. To model this behavior we assume that consumers modify the probability density function $\mathbf{f}_{N_{a}}(n)$ of the daily times of an activity $a$ by:

\footnotetext{
${ }^{2}$ Technically, $\mathbf{f}_{s}(t)$ describes the probability of the activity start time occurring during the day, assuming the activity occurs exactly once. Then, multiple occurrences are handled independently, through $\mathbf{f}_{N}(n)$.
} 


$$
\Delta p(a)=\left(E_{r}(a)-1\right) \cdot s_{e}(a)
$$

where $E_{r}(a)$ is daily energy ratio of activity a given by Equation 3 and $s_{e}(a)$ is the sensitivity to energy reduction. In essence, this formula shows that when the overall energy daily cost for an activity increases, consumers may choose to respond by reducing (and not just shifting) their consumption, while in case the energy ratio is decreased, they may start using the appliances participating in an activity more often.

\subsubsection{Reducing End-Use}

In order to compute the change in case $E_{r}(a)>1$, we first identify the maximum number of times $\left(n_{\max }\right)$ activity $a$ is executed during the day, such that:

$$
f_{N_{a}}(n)=0, \forall n \in N: n>n_{\max }
$$

We then set

$$
f_{N_{a}}^{\prime}(n)= \begin{cases}f_{N_{a}}(n)-\Delta p(a) & \text { if } n=n_{\max } \\ f_{N_{a}}(n) \cdot(1+P \Delta p(a)) & \text { elsewhere }\end{cases}
$$

where $\mathrm{P}$ is given in each case by:

$$
P= \begin{cases}1-f_{N_{a}}\left(n_{\max }\right) & \text { if } n_{\max } \text { is affected } \\ 1-f_{N_{a}}\left(n_{\max }\right)-f_{N_{a}}\left(n_{\max -1}\right) & \text { if } n_{\max } \text { and } n_{\max }-1 \text { are affected } \\ \vdots & \vdots\end{cases}
$$




\subsubsection{Increasing End-Use}

In order to compute the change in case $E_{r}(a)<1$, we slightly change the equations that were defined for the reduction, as we set:

$$
f_{N_{a}}^{\prime}(n)= \begin{cases}f_{N_{a}}(n)-\Delta p(a) & \text { if } n=0 \\ f_{N_{a}}(n) \cdot(1+P \Delta p(a)) & \text { elsewhere }\end{cases}
$$

where $\mathrm{P}$ is given in each case by:

$$
P= \begin{cases}1-f_{N_{a}}(0) & \text { if } n=0 \text { is affected } \\ 1-f_{N_{a}}(0)-f_{N}(1) & \text { if } n=0 \text { and } n=1 \text { are affected } \\ \vdots & \vdots\end{cases}
$$

\section{Implemented Response Models}

This section focuses on the implementation of response models that simulate real-life consumers behavior when motivated by monetary incentives. In the first subsection, a set of general purpose response models based on response analysis of real-life DR programs is defined, while in the second part we present a set of improved response models, fine tuned by using consumer response historical data.

\subsection{Generic Response Models}

Even though the price ratio $r$ is an important factor for the consumers' response behavior, the time shifting sensitivity factor $s_{t}$ is the one that fully describes the shifting capabilities of the activities consumption. Based on results presented by real pilot cases from several DR pilots [13], three different 
types of response models are introduced, that are described in the following sections.

\subsubsection{Price-Based Response}

In this case the sensitivity factor is a constant number:

$$
s_{t}(a, t)=c, t \in\{0,1440\}
$$

Hence, the shifting factor (as described in Equation 5), depends only on the price incentives offered to the consumer.

This response model can describe consumers that are highly sensitive to monetary incentives, trying to reduce consumption in critical peak hours by shifting it to more cost efficient day periods. This scenario is becoming more plausible given the increased penetration of smart appliances in modern households. Price aware electrical appliances are able to shift their operation (automatically or by manual programming) to alternative time periods according to the pricing policy. This type of shifting can result in valley fills, in case of monetary rewards $(r(t)<1)$, or peak reduction, in case of price penalties $(r(t)>1)$, without changing the appliance end-use drastically during the rest of the day. An example of a price sensitive response can be found in Figure $3(\mathrm{a})$.

\subsubsection{Comfort-Based Response}

This response type assumes the consumer attempts to minimize the impact on comfort, by minimum shifting of the appliance operation time. As an example, consider that most consumers can stall their activities for 10 - 15 minutes, whereas the suggestion of a 2 hour delay for some types of activities 
would probably not be accepted. In case of a penalty incentive near the peak index, a percentage of the expected power consumption proportional to the price variation is shifted right before and after the peak window for a temporal distance of $W$, with an inclining effect over distance before peak times and a declining effect over distance after peak times. In case there are additional reward incentives for valley filling, the residual expected power is transferred to the closest valley (in terms of time).

The sensitivity factor for this type of response, given a penalty incentive in the peak price time frame $t \in\left\{T_{\text {start }}, T_{\text {end }}\right\}$, is estimated by the following equation:

$$
s_{t}(a, t)= \begin{cases}\mathcal{N}\left(T_{\text {start }}-d_{\text {mean }}, 2 * W\right) & 0<t<T_{\text {start }}-d_{\text {mean }} \\ 0 & T_{\text {start }}-d_{\text {mean }}<=t<T_{\text {start }} \\ c & T_{\text {start }}<=t<=T_{\text {end }} \\ \mathcal{N}\left(T_{\text {end }}, 2 * W\right) & t>T_{\text {end }}\end{cases}
$$

where $\mathcal{N}(\mu, \sigma)$ symbolizes a Gaussian distribution with mean value of $\mu$ and standard deviation of $\sigma, d_{\text {mean }}$ is the mean duration of the activity under examination, $W$ is the aforementioned shifting window and $c$ is a large constant value.

Depending on the pricing policy applied in the DR program, the shifting may be realized only to earlier times of the day (Figure 3(b) , only to later times of day (Figure 3(c) or on both sides of the peak pricing period (Figure 
$3(d)$.

\subsubsection{Routine-Based Response}

In this response type case scenario, consumers respond to penalty incentives by shifting their activities to the next preferred daytime intervals for this activity. For a consumer that is usually carrying out an activity twice a day (once at noon and once in the evening), penalizing excessive power consumption at noon would often move the activity during the evening. Thus, this shifting behavior is overcharging the smaller peaks of the expected power consumption (Figure 3(e).

To simulate this behavior, the sensitivity factor is set equal to the start time distribution for activity $a$ multiplied by a factor $c$ :

$$
s_{t}(a, t)=c \cdot \mathbf{f}_{s_{a}}(t), t \in\{0,1440\}
$$

\subsection{Computing the sensitivity factor from measurements}

The Smart Grid paradigm and smart metering equipment, that have started being widely used in the recent years, have provided the opportunity to energy-oriented companies to design and deliver new services aiming to raise consumption awareness, such as real-time consumption/production monitoring, energy cost estimation, information on installations' most energy consuming appliances and so on.

In case this type of data is available, there is no need to use the predefined response models presented in Section 2.2, since it is possible to accurately estimate the sensitivity of the installation tenants through measurements. The equation that can be used to estimate the time shifting sensitivity for an 
activity $a$ can be derived directly through Equation 5 and is given by:

$$
s(a, t)=\frac{f_{s_{a}}^{\prime}(t)-f_{s_{a}}(t)}{f_{s_{a}}(t) \cdot \operatorname{shift}(r(t))}, f_{s_{a}}(t) \neq 0
$$

where $t \in\{0,1440\}$ for each minute of the day, $f_{s_{a}}(t)$ is the start time distribution of the activity during the baseline period, $f_{s_{a}}^{\prime}(t)$ is the start time distribution of the activity during the demand response period and $\operatorname{shift}(r(t))$ is given by:

$$
\operatorname{shift}^{\prime}(r(t))= \begin{cases}c_{2} & r(t)<c_{1} \\ \frac{c_{2}}{c_{1}-1} \cdot(r(t)-1) & c_{1}<=r(t)<1 \\ \frac{c_{4}}{c_{3}-1} \cdot(r(t)-1) & 1<=r(t)<c_{3} \\ c_{4} & r(t)>c_{3}\end{cases}
$$

Figure 4 illustrates an example.

Summarizing, the computation of sensitivity factors directly from consumption measurements enables the creation of precise and "personalized" consumer response models. A set of experiments based on this mathematical formulation is presented as a proof of concept in Section 4.1.3.

\section{Experimental Results}

This section discusses the experiments performed using consumption measurements from various installations that participated in real-life DR 
programs. Results illustrate the effectiveness of the proposed small-scale consumer response modeling approach and indicate how precise modeling of the response to monetary incentives can lead to successful decision making, peak power reduction and grid stability.

Raw measurement data were obtained from the EU funded research project $C A S S A N D R A$. CASSANDRA focused on modeling energy market stakeholders in terms of energy profiles and consumption patterns, as well as their interactions, and on increasing the market power of small-scale consumers through Consumer coalitions (Consumer Social Networks - CSNs). The available data were collected from the two pilot case scenarios that were carried out as part of the project evaluation.

Two groups of experiments were performed: (i) Residential Dataset Experiments and (ii) Commercial Dataset Experiments. For the assessment of our methodology, three different evaluation tests were realized: (a) activity modeling evaluation to indicate the accuracy of the individual small-scale consumer activity models extracted from installation consumption measurements (as described in authors' previous work [12]), (b) response modeling evaluation, to examine how well the proposed generic models predict consumer response and (c) simulation of response models computed from measurements (only for the residential dataset), to measure the effectiveness of the data-driven modeling approach presented in section 3.2. Figure 5 depicts the methodology that was followed. Details for each set of experiments is discussed next.

It should be mentioned that direct comparison with other methods presented in the bibliography was not possible due to several reasons: (i) the datasets used in each case are not publicly available, (ii) the goals and evalua- 
tion metrics are different, and (iii) other methods require more input data than the electricity consumption measurements used by our approach (occupancy data, demographics, utility statistical data etc.). Thus, it was impossible to implement/replicate these approaches for the datasets that were available for the evaluation of the present work.

\subsection{Residential Dataset}

The first pilot case took place at a multi-residential building in Lulea, Sweden [14]. This building consisted of 32 apartments and 10 common areas. Measurements of active and reactive power, at one-minute intervals were collected from those installations from January 2013 to February 2014, split in three time periods:

1. Baseline period (January 2013-May 2013): the pricing policy was fixed and the tenants followed their normal consumption behavior.

2. Feedback period (June 2013-October 2013): the same pricing policy was retained, but the tenants were informed of their consumption behavior, as well as of the other tenants' overall consumption, at the end of each month.

3. Demand Response period (November 2013-February 2014): monetary incentives, equivalent to a new pricing scheme, were provided to the tenants. This new scheme included some peak pricing periods, encouraging tenants to respond by shifting and/or reducing their consumption. 


\subsubsection{Residential Dataset Activity Model Experiments}

The first stage of model evaluation is the comparison between simulated and actual baseline power consumption, in order to be sure that we have the appropriate baseline small-scale consumer models to apply the proposed response models. To this end, a set of small-scale consumer models, one for each apartment under examination, was extracted by applying the activity

modeling methodology (presented in authors' previous work [12]) on the available baseline measurements. It should be noted that the common areas of the residential building were omitted from the experimental procedure, since their consumption patterns diverged significantly from the "small-scale" consumers' patterns that this paper studies.

In order to evaluate the precision of our end-use models for each installation four different metrics were employed. All the metrics presented below can be easily calculated from the measurements time series provided for analysis:

- Root Mean Square Error (RMSE)

$$
R M S E=\sqrt{\frac{\sum_{i=1}^{n}\left(v_{i}-p_{i}\right)^{2}}{n}}
$$

where $n$ is the number of time series samples, $v_{i}$ is the observed value of energy consumption for each time step $i$ and $p_{i}$ is the respective predicted value.

- Symmetric Mean Absolute Percentage Error (SMAPE)

$$
S M A P E=\frac{1}{n} \sum_{t=1}^{n} \frac{\left|A_{t}-F_{t}\right|}{A_{t}+F_{t}} 100 \%
$$


where $A_{t}$ is the expected power extracted from the real measurements for the 15-minutes window around $t$ minute of the day and $F_{t}$ is the simulated power for the same window. Given the tolerance levels of the transmission and distribution segments of the power grid, a 10-15\% SMAPE is considered acceptable. Furthermore, the goal of the proposed framework is not focused on consumption or activity forecasting but on consumer activity and response modeling for demand response or consumption analysis applications.

- Mean Daily Energy Error (MDEE)

$$
M D E E=\sum_{i=1}^{n} \frac{\left|v_{i}-p_{i}\right|}{v_{i}} 100 \%
$$

where $n, v_{i}$ and $p_{i}$ are defined as in the RMSE metric.

- Jensen-Shannon Distance (JSD)

$$
J S D(P \| Q)=\sqrt{\frac{1}{2} D_{K L}(P \| M)+\frac{1}{2} D_{K L}(Q \| M)}
$$

where $M=\frac{1}{2}(P+Q)$ and $D_{K L}(P \| M)$ is given by the Kullback-Leibler Divergence equation:

$$
D_{K L}(P \| Q)=\sum_{i} \ln \left(\frac{P(i)}{Q(i)}\right) P(i)
$$

In this work, JSD is used mainly for comparison of prediction accuracy between the predefined response models and the models that are computed from measurement data (Section 4.1.3). 
For the first pilot dataset, the baseline consumption of the apartments was analyzed and an automated disaggregation process was used, which was introduced in [15]. This way, we were able to extract the individual appliances' consumption specifications and their switching events, enabling us to build our activity models. Table 1 presents the results from the residential pilot apartments for the activity model experiments. In addition to the similarity metrics defined above, the Real and the Simulated Mean Daily Energy Consumption (RMDE and SMDE respectively) measures are included in the table, showing how well the models approached reality. The results that show significantly good fitting with the real data are presented in bold.

Given the complexity of the consumer models, one may easily notice that most of the apartments were modeled pretty accurately, meaning small energy and percentage errors. The average SMAPE value for all apartments in this set of experiments was $8.52 \%$, while the respective standard deviation was $2.78 \%$. There are some rare cases where the complexity of the appliances within an installation increased SMAPE (up to 13\%), nevertheless within acceptable error limits for the proposed consumer modeling context. Furthermore, the MDEE values were also low $(\sim 7.61 \pm 5 \%)$. In Figure 6 illustrates the the simulation results of an example installation in fine time granularity (one minute period). The figure show that the proposed model provide very accurate peak time index forecasting and closely follow the trends of the real average power.

\subsubsection{Residential Dataset Response Model Evaluation}

The second set of evaluation tests assessed the response models generated by employing the methodology presented in Section 2 . In order to evaluate 
the different response model types, the various shifting operations outlined of sections 2.2 and 2.3 were applied to the bottom-up models of the small-scale consumers created for the first set of experiments. The assessment of the models was performed by comparing the real measurements from the demand response period with the simulated response models, after applying the same monetary incentives as the real pilots (rewards and penalties). Thus, for the realization of these experiments, two different pricing schemes were defined:

1. A baseline pricing scheme, $p_{\text {base }}(t)$, with fixed price/ $k$ Wh all day long.

2. A critical peak pricing scheme, $p_{\text {new }}(t)$, where the peak periods of the day have increased price, while the rest have slightly reduced prices, in order to incentivize shifting away from on-peak to off-peak periods.

Figure 7 provides an overview of the pricing schemes utilized in the baseline and the DR period of the residential pilot. It is important to mention that (both pilot cases) DR programs under examination were implemented through pricing schemes with increased energy cost. This way the participants of the DR program were encouraged to reduce their overall consumption (mostly at the peak pricing periods, but on the other hours of the day as well) in order to maintain a low billing cost.

In the residential pilot case, given the 1 year difference between the baseline and the DR period, some of the apartments became invalid for our model evaluation purposes. More specifically:

- Some tenants were absent during the baseline period.

- Some the tenants changed their apartments' electrical equipment. 
- In certain apartments the consumption patterns changed significantly, indicating a change of tenant or a major change in behavior for reasons unknown to us.

In order to draw meaningful conclusions during the evaluation procedure of the response models, these apartments were excluded from the response modeling evaluation experiments (since the response models for these apartments were considered obsolete for the DR period).

In order to assess the results of the response models, their similarity with the actual response measurements was estimated. Apart from the metrics used in the previous subsection (RMSE, SMAPE, MDEE, JSD), three more metrics were used for the comparison with the real response measurements from the installations:

- Peak Reduction: This metric shows the load reduction over the peak periods of the day as a percentage.

$$
\Delta_{\text {peak }}=\frac{\sum_{i} \mathcal{E}_{\text {baseline }}(i)-\sum_{i} \mathcal{E}_{\text {response }}(i)}{\sum_{i} \mathcal{E}_{\text {baseline }}(i)} 100 \%
$$

where $i \in\{0,1440\}$ the subset of the minutes of the day within the critical peak pricing period, $\mathcal{E}_{\text {baseline }}$ is the mean daily energy vector for the baseline measurements and $\mathcal{E}_{\text {response }}$ is the mean daily energy vector for the demand response measurements. Positive values imply that there was a reduction in consumption, while negative values signify an increase in consumption during the same period.

- Off-Peak Reduction: This metric specifies the load percentage reduction 
over the off-peak periods of the day. It is defined accordingly:

$$
\Delta_{\text {off-peak }}=\frac{\sum_{i} \mathcal{E}_{\text {baseline }}(i)-\sum_{i} \mathcal{E}_{\text {response }}(i)}{\sum_{i} \mathcal{E}_{\text {baseline }}(i)} 100 \%
$$

where this time $i \in\{0,1440\}$ the subset of the minutes of the day outside the critical peak pricing period.

- Overall Reduction: This metric shows the overall daily reduction:

$$
\Delta_{\text {overall }}=\frac{\sum_{i=1}^{1440} \mathcal{E}_{\text {baseline }}(i)-\sum_{i=1}^{1440} \mathcal{E}_{\text {response }}(i)}{\sum_{i=1}^{1440} \mathcal{E}_{\text {baseline }}(i)} 100 \%
$$

Table 2 presents the results from individual residential apartments comparison between real and simulated response. The first section of the table shows the results from the real measurements analysis, representing the reduction or shifting by the pilot case consumers as they were actually estimated from the available measurements. The second part of the table shows the attributes of the shifting model that best fit the actual response period measurements, namely the type of response model used (as defined in 3.1), the Time Shifting Sensitivity factor (TSS) in [0,1] and the Energy Ratio Sensitivity factor (ERS) with three different levels (Low, Medium, High). The third part of the table shows the similarity metrics (RMSE, SMAPE, MDEE, JSD) comparing real and simulated response, while the forth part presents the reduction metrics in comparison with the real baseline measurements, trying to follow closely the actual response reduction. The results that show significantly good fitting with the real response data are presented in bold. 
From the results presented, it is obvious that some tenants were actively trying to reduce their overall consumption as expected (e.g. apartments $4,14,29)$. These consumers were successfully simulated by models that have medium or high ERS. At the same time, other tenants preferred time shifting from reducing their daily consumption (for example apartments $2,7,8)$. In some of those cases, the large consumption shifting from the on-peak periods to the off-peak ones in combination with a small increase in the consumption during off-peak periods resulted in an increase in the overall daily consumption. These tenants were simulated mostly by a low ERS factor and a medium or large TSS factor. Finally, there was one apartment that the tenants didn't reduce the consumption at any period of the day (apartment 20), which was simulated best from a model with no response, as it should have. It should be noted that the best fitted exported response models were based on comfort or routine, meaning that the tenants of the households were susceptible to change their behavior, but without having to change their habits or to decrease their comfort significantly. Figure 8 shows the comparison chart between the real and the simulated response of an apartment.

In summary, the results from the residential pilot apartments show that SMAPE stayed in low levels (10.97 $\pm 5.14 \%)$, as well as MDEE (3.66 $\pm 3.94 \%)$. This means that we succeeded in portraying the actual installation response with the generalized models introduced in Section 3.1. From all apartments analyzed, only two apartments had SMAPE over 20\%, leading to deduce that, in this case, our response models were not able to follow the actual response trends accurately. 


\subsubsection{Response model simulations computed from measurements}

This final set of evaluation tests for the residential pilot case aims at evaluating the proposed approach for computing the sensitivity factor from DR measurements, as described in section 3.2. Unfortunately, the small duration of the DR period of the pilot case did not allow a full training/test set evaluation cycle. Instead, we restricted ourselves to presenting a proof of concept by estimating the sensitivity factor using the entire DR period and then showing how the newly computed response models fit the data.

In this experimental set, we were trying to improve the results of the previous response experiments set. The new results are presented in Table 3 , using the same metrics as above so that they can be easily compared with the ones from the previous approach. To help the reader with the comparison and outline the effectiveness of the analytical approach, the similarity metrics results that were improved in comparison with the previous set are presented in bold.

It can be seen that, in all cases, the analytical approach resulted in better SMAPE and JSD, from slightly better up to $300 \%$ in some cases. Overall, the results are better than the ones provided from the predefined models of the previous set (SMAPE: $6.86 \pm 2.58 \%$ and MDEE: $3.26 \pm 2.90 \%$ respectively). This means that the new models follow more closely the actual response of consumers in fine time granularity, leading to more realistic and flexible models. It should be mentioned that in some cases the absolute error on the reductions (peak, off-peak and overall) was ameliorated, but in all cases it stayed within acceptable limits, meaning that the results are reliable and useful. Figure 9 illustrates an example of the improved response models with 
the use of the analytical approach, in comparison with the generic approach of the previous section. The same apartment was selected so that an immediate comparison between the two approaches is possible.

\subsection{Commercial Dataset}

The second pilot took place at a commercial center near Milan, Italy [16]. This mall contains many shops, a swimming pool and large common areas. In this case, 11 businesses of various types (i.e. gym, jewelry, beauty shop etc.) actively participated in the DR program of the pilot. Their measurements of active and reactive power, at one-minute intervals were collected from the Building Management System of the commercial center.

This pilot was also separated in three periods with the same characteristics as the ones in the previous pilot: (i) baseline period (September 2012 - January 2013), (ii) feedback period (September 2013 - January 2014) and (iii) demand response period (February 2014).

\subsubsection{Commercial Dataset Activity Model Experiments}

For the first stage of the second pilot case evaluation, the small-scale consumer models simulating the consumption behavior of the shops from the commercial center were assessed. This time, the installation models were created manually using the $C A S S A N D R A$ platform ${ }^{3}$, which serves as a friendly and comprehensive GUI for building analytical activity and appliance models. The participating shopkeepers administered the specifications of the shops' electrical equipment, as well as their daily schedule of appliances end-use. Based on the information provided, detailed activity models were

\footnotetext{
$\sqrt[3]{\text { http://www.cassandra-fp7.eu/page/cassandra_software_platform }}$
} 
created, matching closely the mean daily energy consumption as observed from the baseline measurements of the shops. The metrics used for evaluation were the same as with the residential pilot dataset.

Table 4 presents the results from the shops in the mall. One may easily notice that the shops that have a tight, regular schedule were very accurately portrayed by the extracted activity models. However, there were also some individual shops with more complex schedule or specialized electrical equipment which led to less precise models (e.g. shop 6, which is a Laundry Service, exhibits SMAPE of $\sim 16 \%$ ). Overall, the results are within acceptable error range (SMAPE: $6.03 \pm 3.85 \%$ and MDEE: $0.59 \pm 0.48 \%$ respectively) for the activity modeling context of the work. Figure 10 compares the actual and estimated power consumption for a representative shop example.

\subsubsection{Commercial Dataset Response Model Evaluation}

Before citing the results, it is important to present the pricing schemes utilized in the baseline and the DR period of the commercial pilot (Figure 11). This DR program also uses an increased energy price (i.e. Energy Ratio $E_{r}>1$ for most activities), so that the shopkeepers are encouraged to reduce their overall consumption, if this is permitted by operational restrictions. The metrics used for evaluation were the same as with the residential pilot dataset.

Moving on to the results, Table 5 shows that the extracted shop response models generated very accurate results as well. SMAPE values were estimated at $\sim 9.83 \pm 5.00 \%$ and MDEE was also at the same low levels as before $(0.59 \pm 0.48 \%)$, meaning that we can accurately portray the actual installation response in this pilot as well. Figure 12 shows the comparison chart between the real and the simulated response of a selected shop. 
As far as the general response behavior of the shops is concerned, there were some shops that couldn't reduce their consumption at all due to operational limitations, while other shops reduced their consumption significantly, showing great response/reduction potential. For commercial installations, routinebased and price-based responses were more common, since the monetary incentives and the tight time schedule played a more important role in shop managers' consumption patterns. This fact was successfully identified by the extracted models. As in the previous pilot, certain shops with specialized appliances and machinery (pool, gym, etc.) were not as accurately portrayed (having SMAPE slightly over 10\%).

\subsection{Discussion}

The activity model evaluation phase employed single installation measurements in order to: (a) assess the effectiveness of the proposed activity modeling approach in simulating small-scale consumers, (b) directly compare real and predicted energy consumption and, finally, (c) evaluate the accuracy of the activity models in fine temporal granularity.

Judging by the better performance of the second pilot's installations models, it seems that having an appliance specification list, in addition to the detailed schedule of the shops leads to more accurate models that the ones provided by the disaggregation. Thus, in the first pilot we have less accurate models, as a result to the abundance of electrical appliances in each house and an arbitrary, varying schedule of the tenants living in.

As far as the response model evaluation is concerned, two aspects were evaluated: (a) the time shifting capabilities of consumption from the peak to the off-peak periods of the day, showing the accuracy of the time shifting 
models and, (b) the impact of a non-neutral pricing scheme (i.e. one where the total electricity price increases), where the consumers are incentivized to reduce the overall consumption throughout the day.

Summarizing, the error level of the response modeling experiments is more than adequate to characterize and/or group consumers and support the design of effective DR programs. It should be noted that in some cases the achieved SMAPE and JSD values (which are more fine grained metrics) are even satisfactory for consumption prediction problems. Overall, the small-scale consumer models extracted from the measurements portray accurately the installations under examination.

Another practical aspect of the proposed work was revealed by the fact that the DR period took place one year later than the baseline period. Some of the installations of the residential dataset had undergone significant changes during this time (in terms of occupancy profile, consumer behavior or appliance equipment), which affected the validity of the response models for the DR period. In practice stakeholders can easily tackle this issue by (i) continuously collecting data to update their activity and response models and (ii) by continuously evaluating, via the proposed sensitivity factor (Section 2.1.2), the sensitivity and response behavior of individual consumers.

The results of all the scenarios examined indicate that a significant decompression in the peak area is feasible without having to increase prices too much or heavily penalize consumers who choose not to accept the end-use change. Additionally, one should mention that this set of experiments also reveals a significant benefit of the presented bottom-up modeling approach: having detailed small-scale models allows for sending different incentive schemes to 
each consumer agent (or groups of them), thus leading to finer control of the power curve. This intuition should be further explored in future work.

In conclusion, these simulations illustrate how the proposed response model methodology, along with the proposed activity training mechanism, can be used to model and predict the consumer reactions for a range of DR scenarios involving a variety of incentives/pricing schemes and different consumer responses, providing effective decision support, both from the supplier and the demand side. Examples of such decision support applications of the proposed methodology are (i) aggregators, wishing to optimize their portfolio management, (ii) DSOs, wishing to eliminate high peaks in demand, (iii) municipalities, companies, or organizations aiming at lowering the environmental impact and cost of electrical energy consumption, (iv) individual consumers, wishing to select their energy provider or optimally adapt their consumption to reduce household electricity costs. In addition, these experiments indicate that even though the demand of each individual small-scale consumer is very small in the context of the distribution system, change in consumption behavior of the individual consumers can aggregate and have significant impact.

\section{Conclusion and Future Work}

Current work presents detailed bottom-up response models for simulating the consumption behavioral change of small-scale consumers under DR program policies. By providing measurements from installation-level power consumption data, detailed activity models are initially built, which can simulate the observed appliance end-use. Then, a set of response models 
is generated that builds on these activity models to predict changes in consumption due to the individuals' change of behavior, simulated via monetary incentives and taking into account monetary sensitivity and comfort. Finally, in case the necessary input data are available, an analytical way to compute the sensitivity of the consumer is provided, allowing the response behavior to be modeled for each consumer individually and with greater accuracy. Using the proposed approach, an Energy Provider or DSO can map the observed power consumption to consumer activities and therefore model and simulate a wide range of DR scenarios for load shaping.

A number of experiments were carried out based on measurements that were collected from apartments of a residential building in Sweden and from shops in a commercial center in Italy as part of the CASSANDRA research project. The result of these experiments illustrated the accuracy and applicability of our implemented activity and response models. An interesting observation is that even though the proposed generic response models were able to simulate with adequate accuracy the actual consumer response, the result were significantly improved when available data enabled the computation of the sensitivity parameter. This is quite important, since the emergence of Smart Grids allows Energy Providers to collect large amounts of detailed measurement data from their consumers.

Results presented in this paper are promising, however there is room for future improvement. Activity models can also become context-dependent, e.g. different models for working/non-working days, different seasons of the year etc. It is also possible to examine the use of a different type of shifting functions (sigmoid, parabolic) and have the parameters trained from available 
data. Also, more factors may be introduced in the consumer sensitivity estimation such as environmental awareness and social factors. Another aspect worth exploring is the possibility of having dynamic pricing schemes that may change over time, which would require an automated procedure that would retrain the models when the pricing policy changes. Finally, as more Smart Grid measurement data become available, the models can be encapsulated to multi-agent systems with several agents playing the role of the installation tenants in order to experiment with different Demand Response incentives applied at varying community sizes (such as neighborhoods or cities).

\section{Acknowledgments}

This work was supported in part by the EU funded research project CASSANDRA (FP7-ICT-288429).

\section{References}

[1] M. Albadi, E. Elsaadany, A summary of demand response in electricity markets, Electric Power Systems Research 78 (11) (2008) 1989-1996.

URL http://linkinghub.elsevier.com/retrieve/pii/ S0378779608001272

[2] Eia, Electric power annual 2009, Energy 0348.

URL http://www.eia.doe.gov/cneaf/electricity/epa/epa.pdf

[3] B. Kladnik, A. Gubina, G. Artac, K. Nagode, I. Kockar, Agent-based modeling of the demand-side flexibility, in: Power and Energy Society General Meeting, 2011 IEEE, IEEE, 2011, pp. 1-8. 
[4] A. Kashif, X. H. B. Le, J. Dugdale, S. Ploix, Agent based framework to simulate inhabitants' behaviour in domestic settings for energy management., in: International Conference on Agents And Artificial Intelligence, 2011, pp. 190-199.

[5] S. Bahrami, M. Parniani, A. Vafaeimehr, A modified approach for residential load scheduling using smart meters, in: Innovative Smart Grid Technologies (ISGT Europe), 2012 3rd IEEE PES International Conference and Exhibition on, IEEE, 2012, pp. 1-8.

[6] S. Gyamfi, S. Krumdieck, Scenario analysis of residential demand response at network peak periods, Electric Power Systems Research 93 (2012) 32-38.

[7] W. Jia, C. Kang, Q. Chen, Analysis on demand-side interactive response capability for power system dispatch in a smart grid framework, Electric Power Systems Research 90 (2012) 11-17.

[8] I. Stoyanova, M. Marin, A. Monti, Characterization of load profile deviations for residential buildings, in: Innovative Smart Grid Technologies (ISGT Europe), 4th IEEE PES International Conference and Exhibition on, IEEE, 2013, pp. 1-5.

[9] O. Corradi, H. Ochsenfeld, H. Madsen, P. Pinson, Controlling electricity consumption by forecasting its response to varying prices, Power Systems, IEEE Transactions on 28 (1) (2013) 421-429.

[10] E. Karfopoulos, L. Tena, A. Torres, P. Salas, J. G. Jorda, A. Dimeas, N. Hatziargyriou, A multi-agent system providing demand response 
services from residential consumers, Electric Power Systems Research (0) (2014) In Press.

[11] J. Yusta, H. Khodr, A. Urdaneta, Optimal pricing of default customers in electrical distribution systems: Effect behavior performance of demand response models, Electric power systems research 77 (5) (2007) 548-558.

[12] A. Chrysopoulos, C. Diou, A. Symeonidis, P. Mitkas, Bottom-up modeling of small-scale energy consumers for effective demand response applications, Engineering Applications of Artificial Intelligence 35 (0) (2014) 299

- 315. doi:http://dx.doi.org/10.1016/j .engappai.2014.06.015.

URL http://www.sciencedirect.com/science/article/pii/ S0952197614001377

[13] J. Stromback, C. Dromacque, M. H. Yassin, G. E. T. T. VaasaETT, The potential of smart meter enabled programs to increase energy and systems efficiency: a mass pilot comparison short name: Empower demand, Vaasa ETT.

[14] M. Holst, M. Runardotter, E. Törnkvist, C. Diou, C. Dromacque, J. Stromback, R. Grigoriou, Deliverable D6.2 pilot case 2 (consumers network) deployment, Tech. rep., CASSANDRA Project (FP7-288429) (2013).

URL http://www.cassandra-fp7.eu/uploads/files/35_ ICT288429CASSANDRAD6.2_Pilot_case_2_deployment.pdf

[15] A. N. Milioudis, G. T. Andreou, V. Katsanou, K. Sgouras, D. P. Labridis, Event detection for load disaggregation in smart metering, in: Innovative 
Smart Grid Technologies (ISGT Europe), 4th IEEE PES International Conference and Exhibition on, IEEE, 2013, pp. 1-5.

[16] L. Ferrarini, G. Mantovani, M. Parigi, Deliverable D5.2 pilot case 1 (shopping center) deployment, Tech. rep., CASSANDRA Project (FP7-288429) (2013).

URL http://www.cassandra-fp7.eu/uploads/files/34_ ICT288429CASSANDRAD5.2_Pilot_case_1_deployment.pdf 


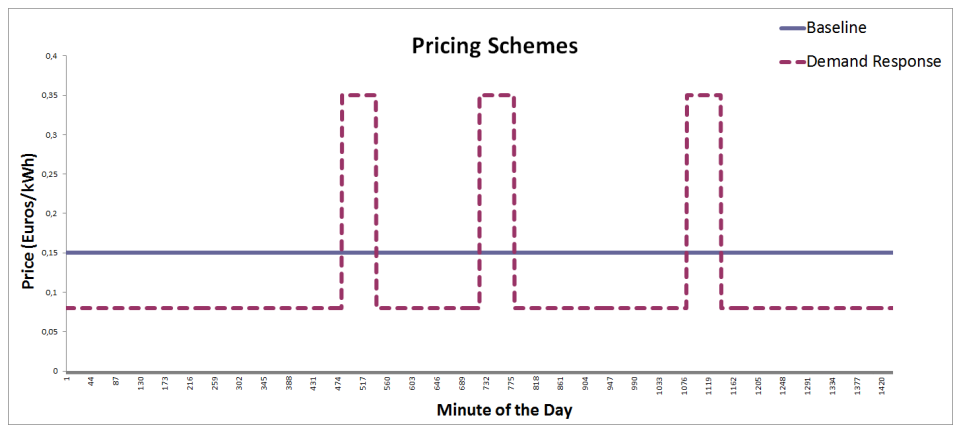

(a)

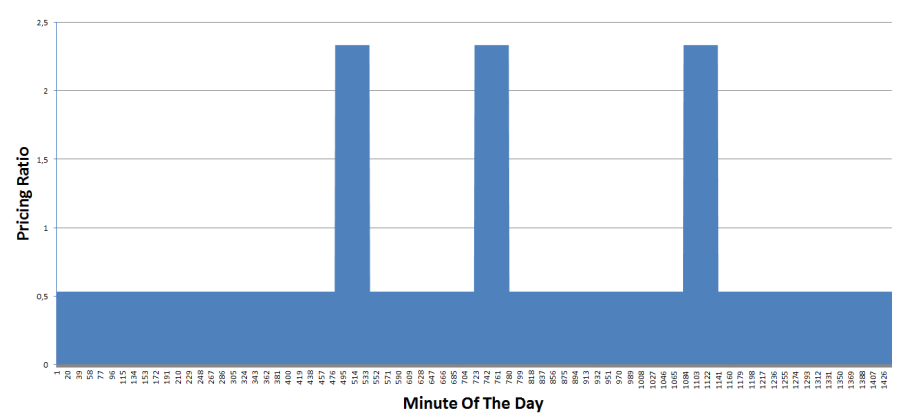

(b)

Figure 1: Example of (a) ToU pricing schemes and (b) their resulting pricing ratio. 


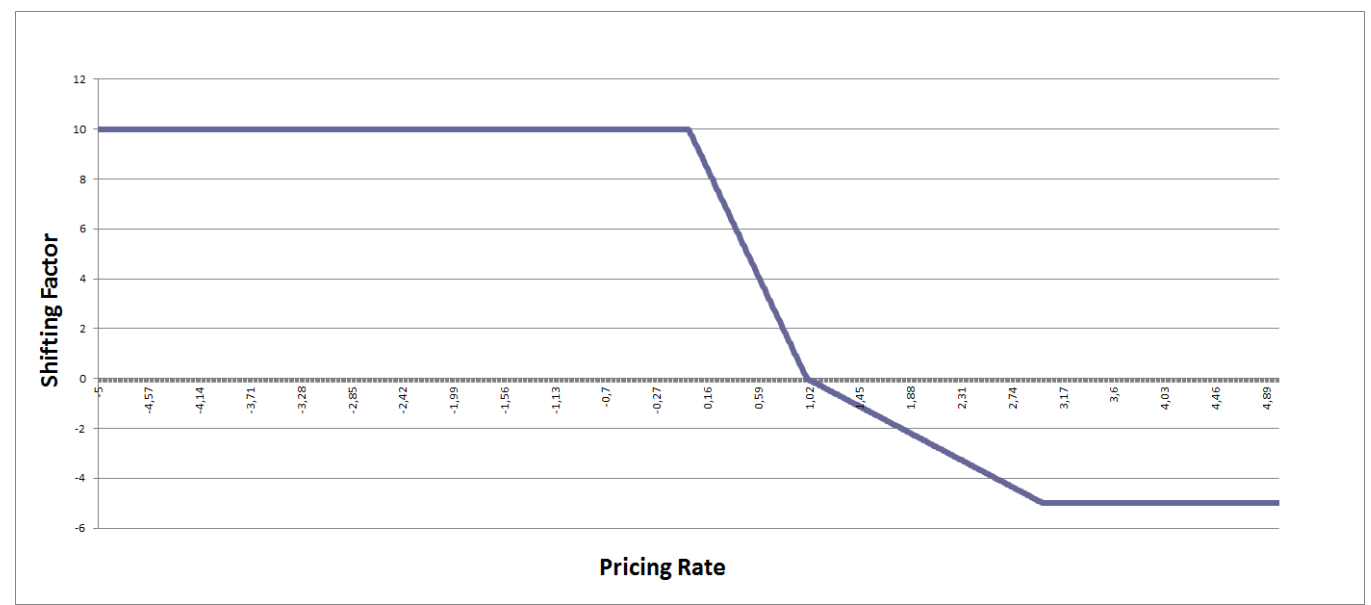

Figure 2: The piecewise linear function used in all our experiments. Parameters are set as: $c_{1}=0, c_{2}=10, c_{3}=3, c_{4}=-5$. 


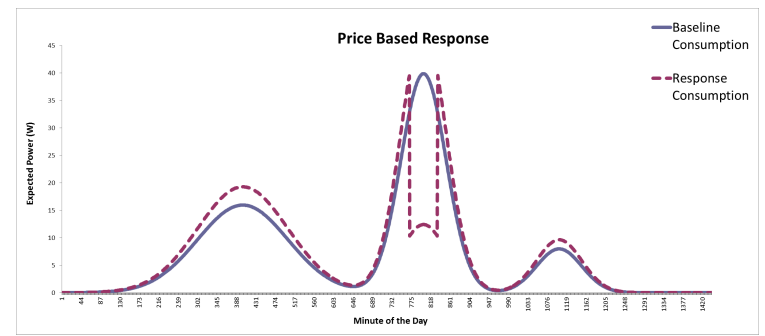

(a) Price-Based

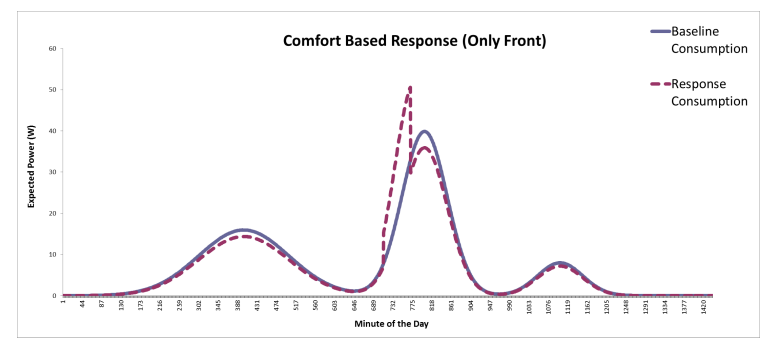

(b) Comfort-Based Front

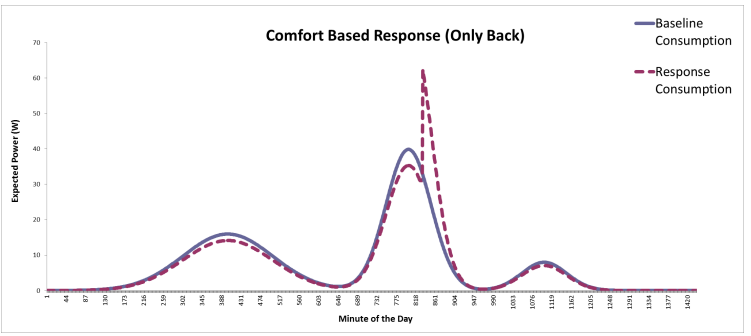

(c) Comfort-Based Back

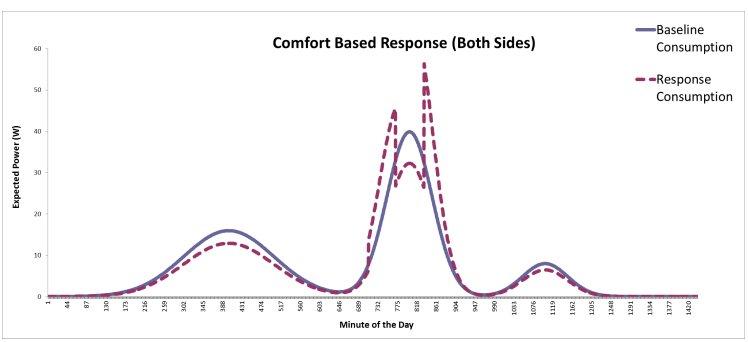

(d) Comfort-Based Both

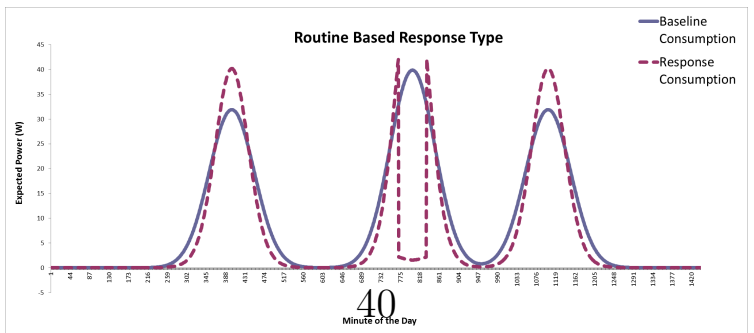

(e) Routine-Based

Figure 3: Example of the generalized response models implemented in this work for a $W$ $=30$ minutes. In this example, a critical peak pricing policy is applied around the time index of the center of the large peak (minutes $775-825$ ) 


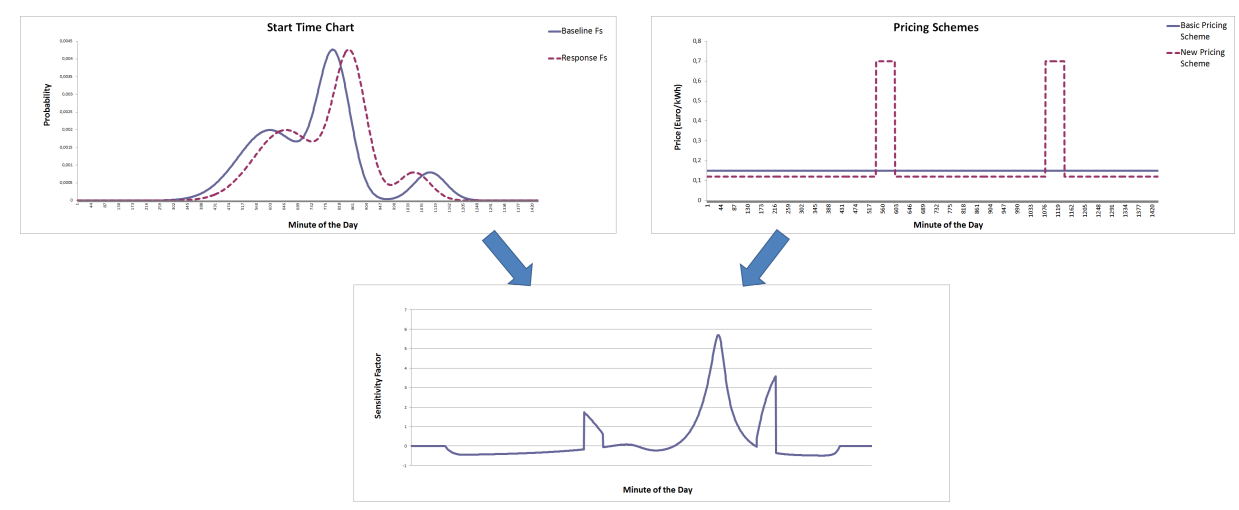

Figure 4: This is an example of the procedure followed in order to compute sensitivity from data. The inputs are the start time probability functions during the baseline and the response periods with the respective pricing schemes, resulting to the analytical model of time shifting sensitivity.

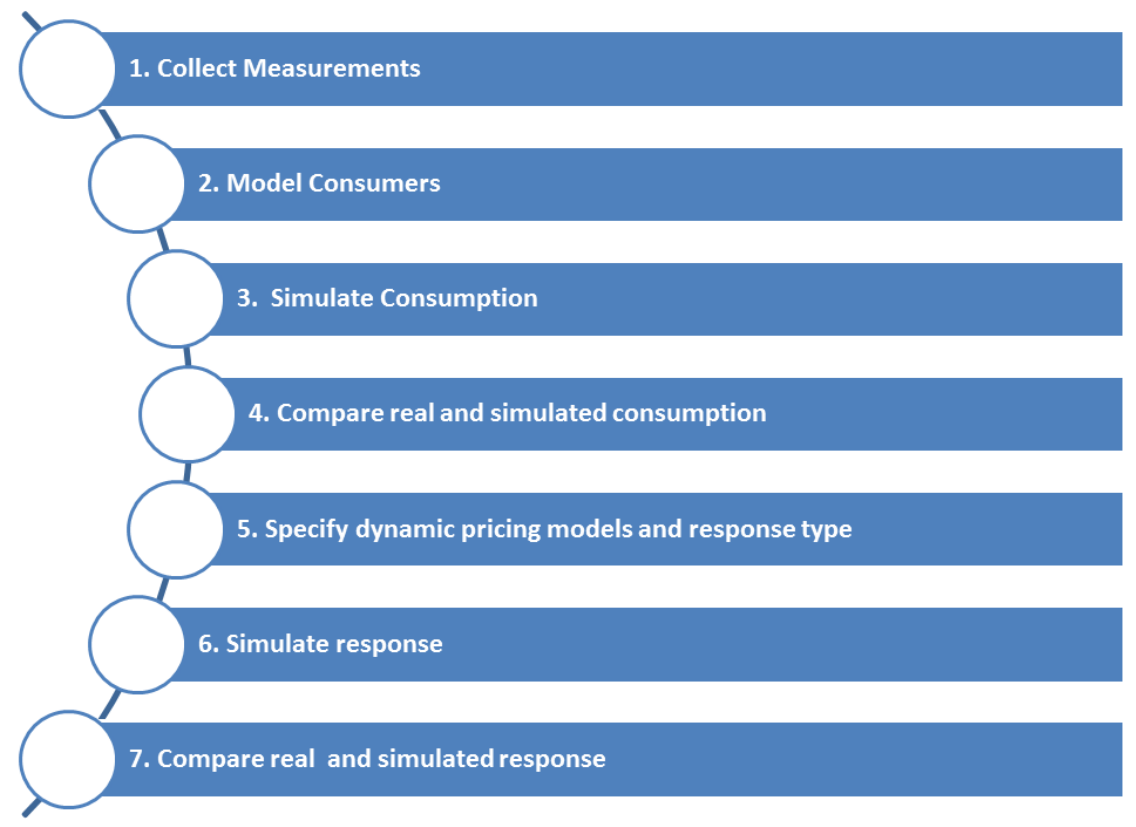

Figure 5: This figure illustrates the procedure followed during the experiments for each installation. 


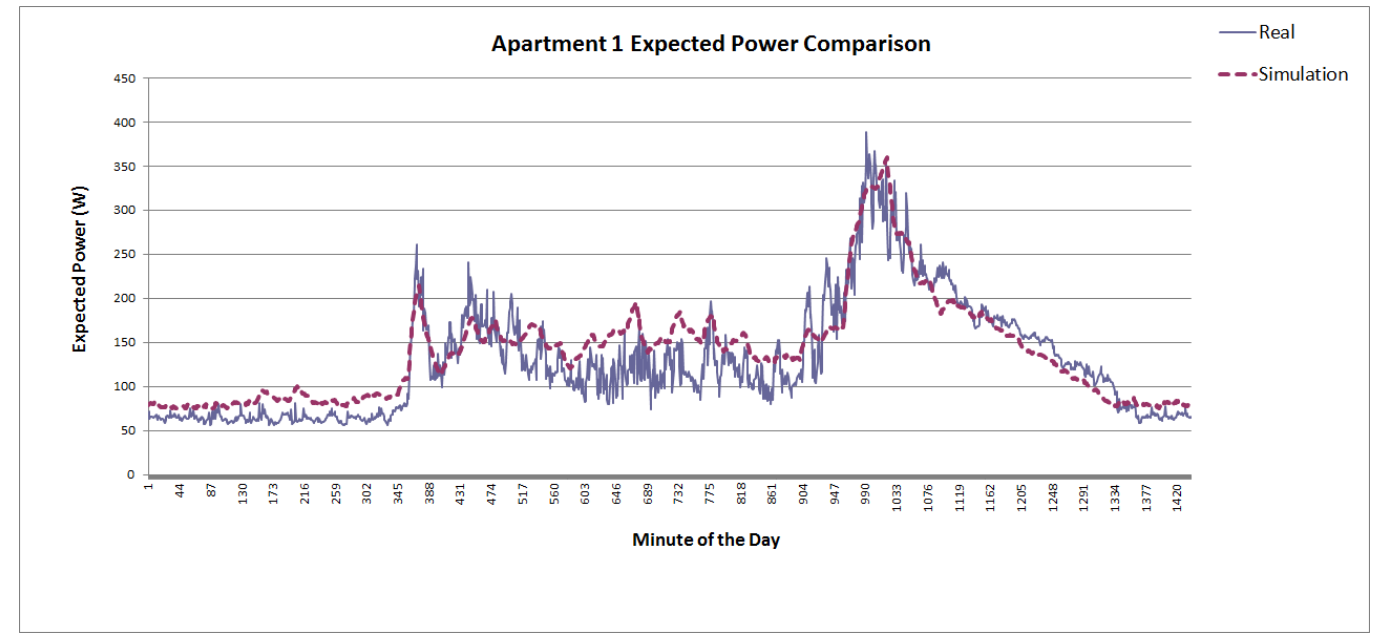

Figure 6: Example of a simulated apartment (Apartment 1) from the residential pilot, compared with its actual baseline measurements.

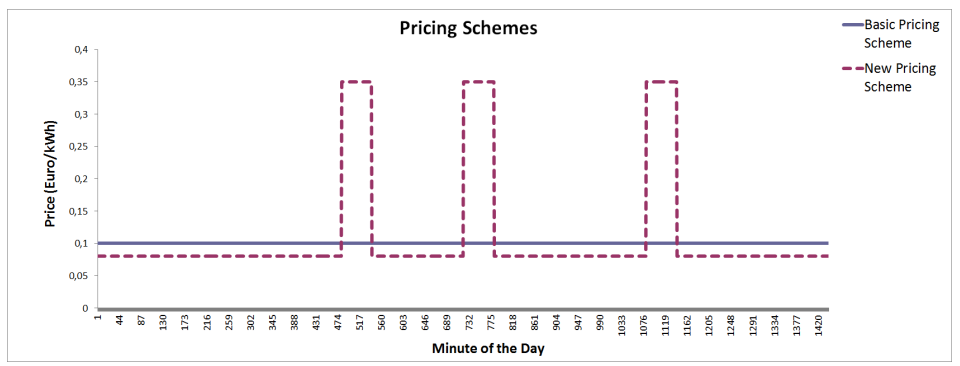

Figure 7: The TOU pricing schemes for the residential pilot case 


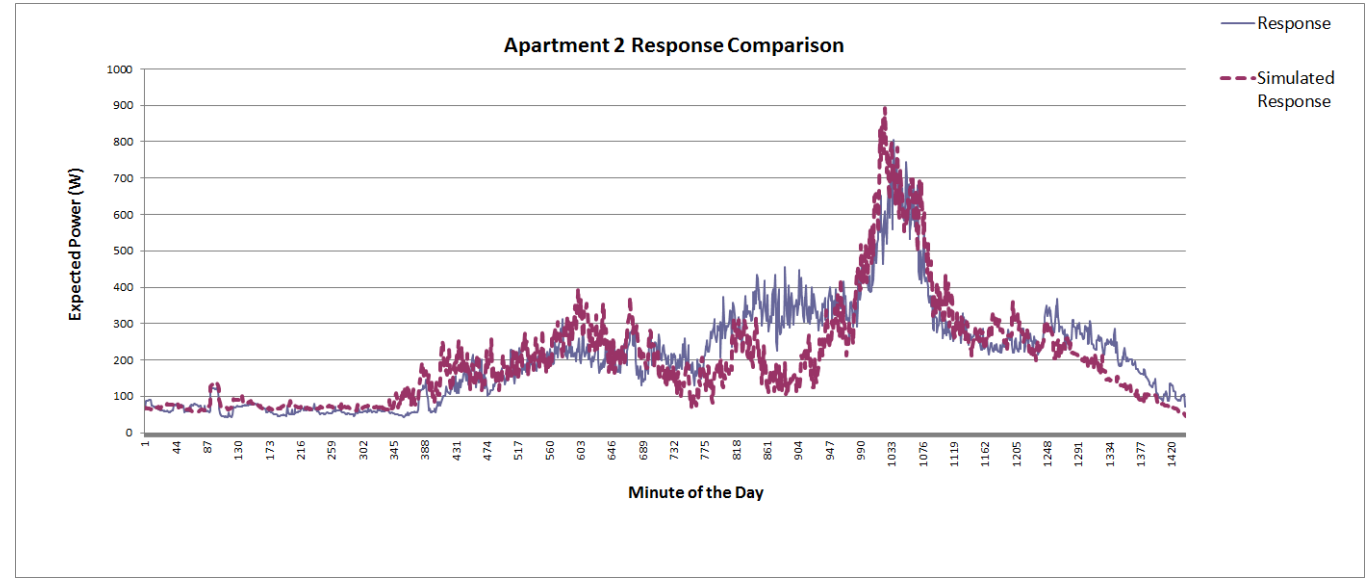

Figure 8: Example of a simulated response from the residential pilot (Apartment 2), compared with the actual response period measurements.

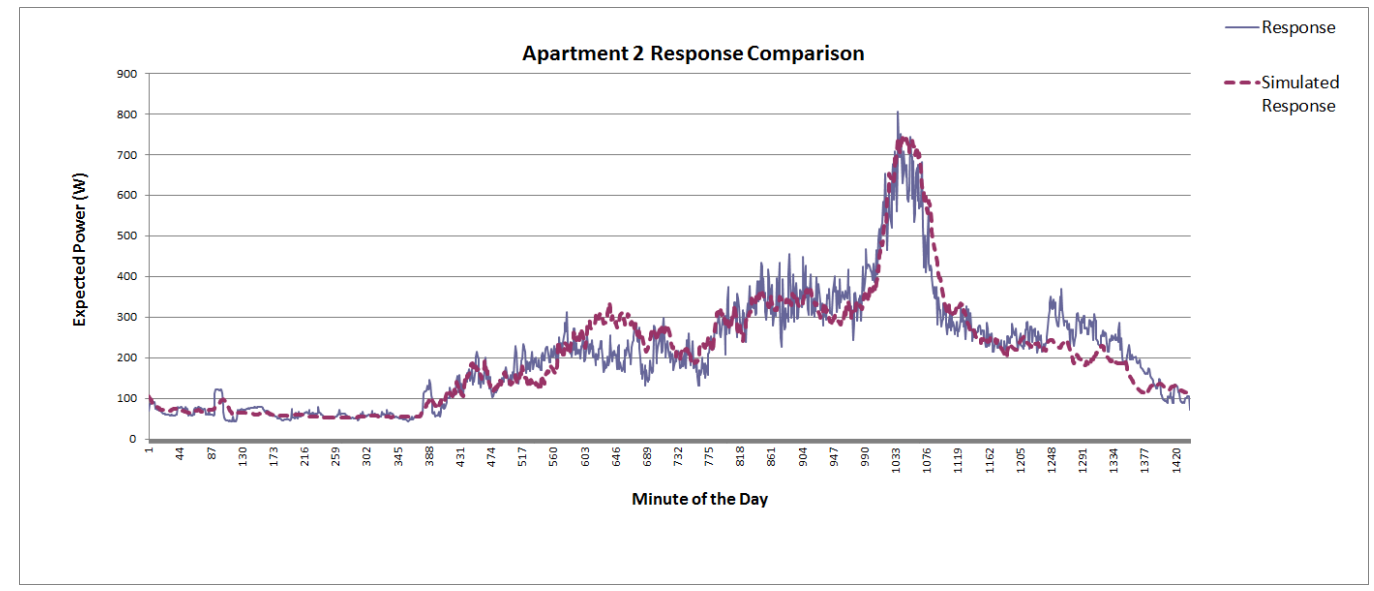

Figure 9: Example of a simulated response from the residential pilot (Apartment 2 again) with the custom sensitivity modeling, compared with the actual response period measurements. 


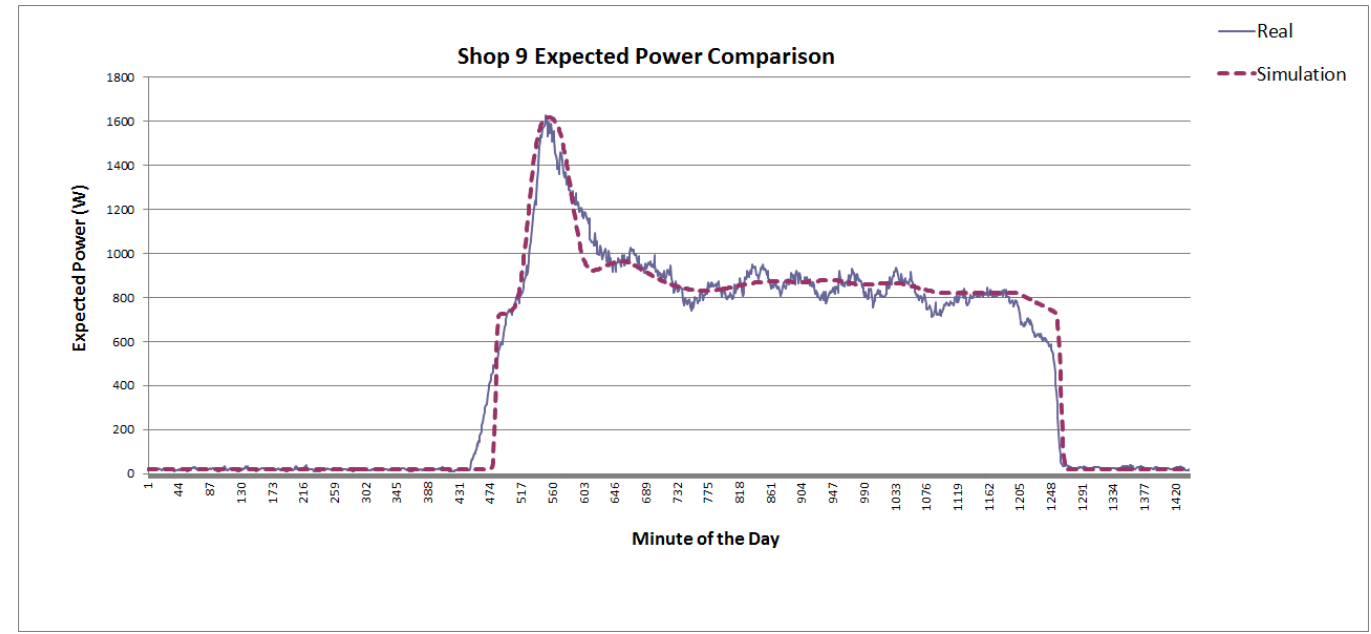

Figure 10: Example of a simulated shop (Shop 9) from the commercial center, compared with the actual baseline measurements.

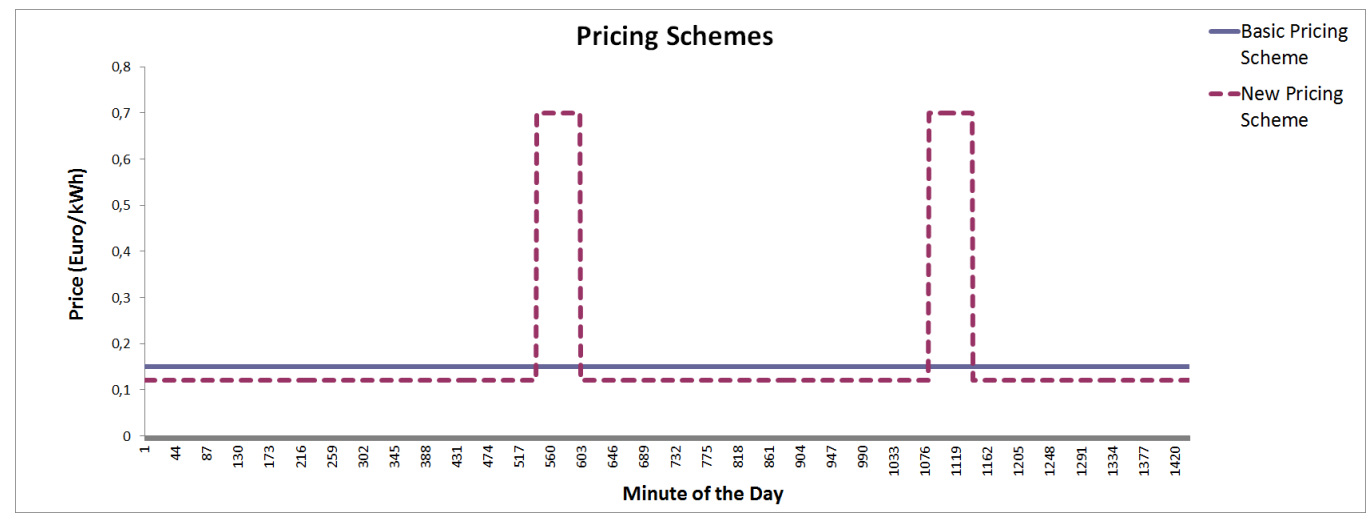

Figure 11: The TOU pricing schemes for the residential pilot case 


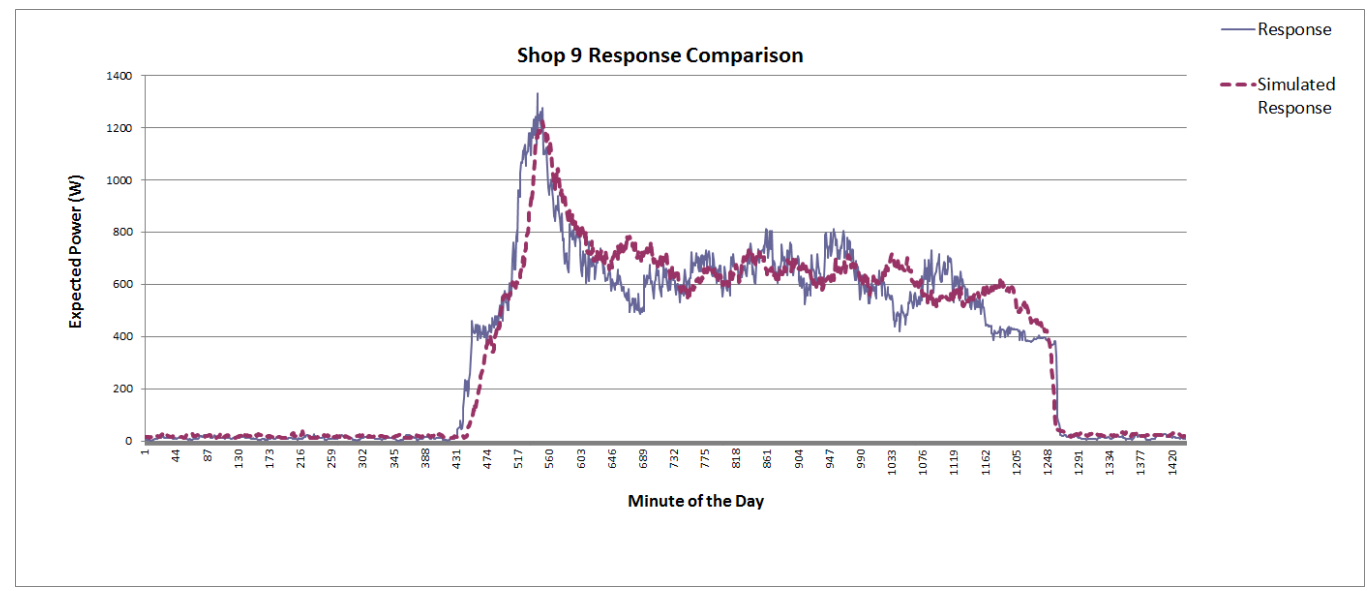

Figure 12: Example of a simulated response from the commercial center pilot (Shop 9), compared with the actual response period measurements. 
Table 1: The activity model simulation results for each apartment of the residential pilot case.

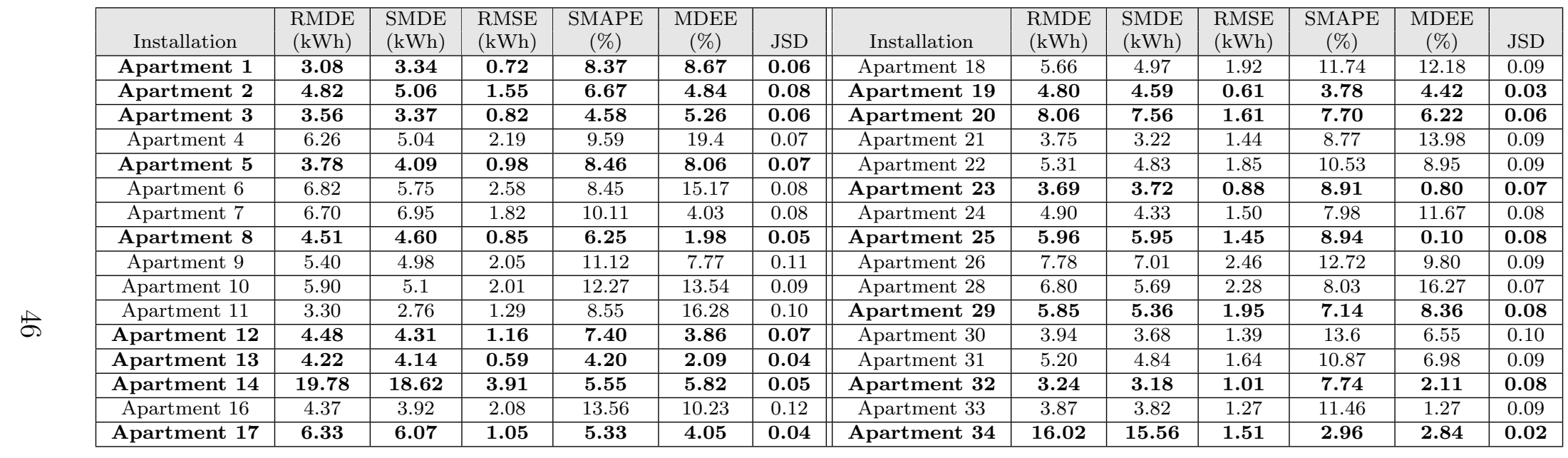


Table 2: Predefined response modeling results for residential pilot apartments.

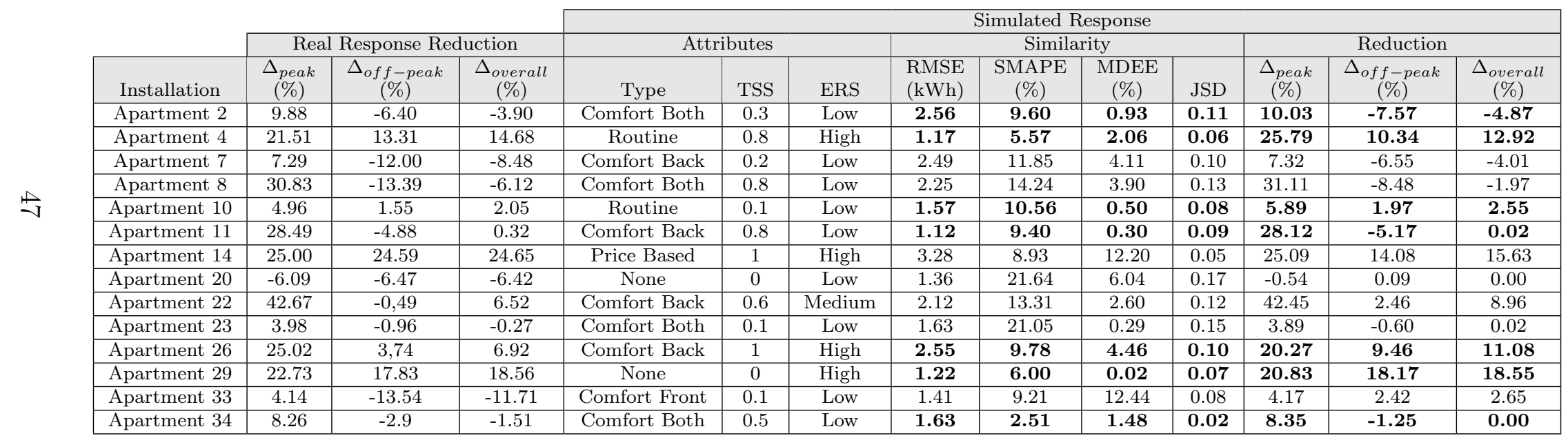


Table 3: Result of the response models with computed sensitivity factor for residential pilot apartments.

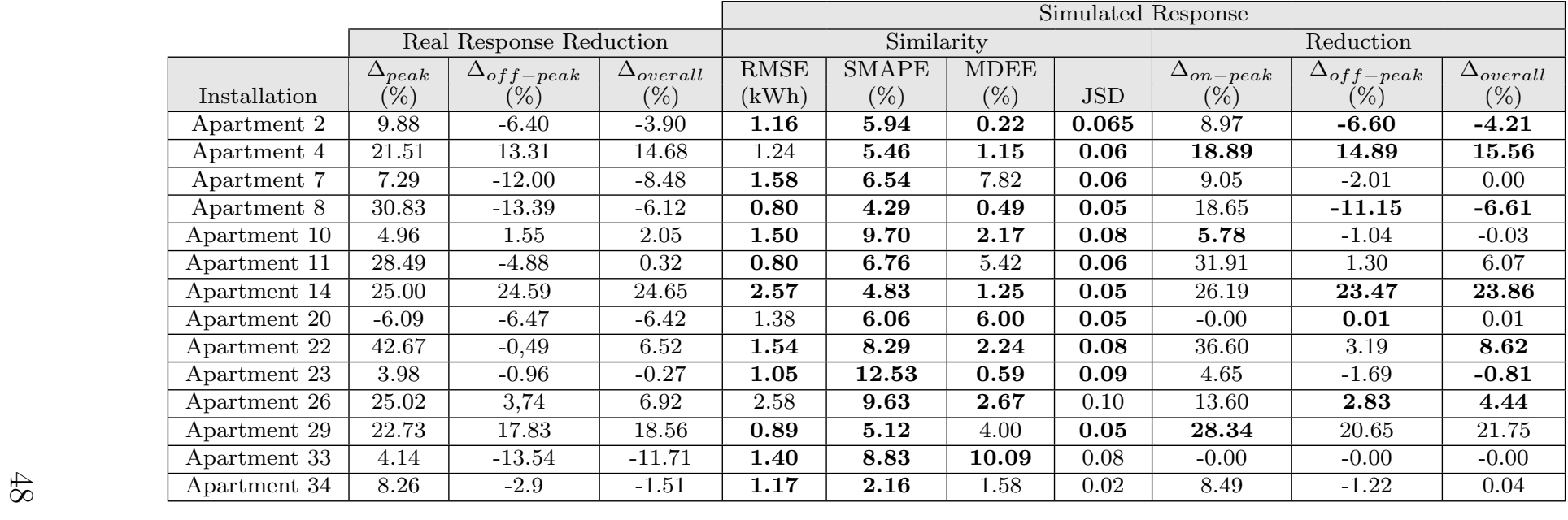

Table 4: The activity model simulation results for each shop participating in the commercial center pilot

\begin{tabular}{|c|c|c|c|c|c|c|}
\hline Installation & $\begin{array}{c}\text { RMDE } \\
(\mathrm{kWh})\end{array}$ & $\begin{array}{c}\text { SMDE } \\
(\mathrm{kWh})\end{array}$ & $\begin{array}{c}\text { RMSE } \\
(\mathrm{kWh})\end{array}$ & $\begin{array}{c}\text { SMAPE } \\
(\%)\end{array}$ & $\begin{array}{c}\text { MDEE } \\
(\%)\end{array}$ & JSD \\
\hline Shop 1 & $\mathbf{1 1 4 . 1 3}$ & $\mathbf{1 1 4 . 8 2}$ & $\mathbf{1 5 . 0 5}$ & $\mathbf{5 . 0 4}$ & $\mathbf{0 . 6 0}$ & $\mathbf{0 . 0 3}$ \\
\hline Shop 2 & $\mathbf{1 0 1 . 6 1}$ & $\mathbf{1 0 1 . 2}$ & $\mathbf{4 . 5 0}$ & $\mathbf{0 . 9 7}$ & $\mathbf{0 . 4 0}$ & $\mathbf{0 . 0 1}$ \\
\hline Shop 3 & 33.37 & 33.27 & 1.44 & 6.56 & 0.30 & 0.03 \\
\hline Shop 4 & $\mathbf{3 7 6 . 1 0}$ & $\mathbf{3 7 6 . 3 8}$ & $\mathbf{2 5 . 1 6}$ & $\mathbf{3 . 7 3}$ & $\mathbf{0 . 0 7}$ & $\mathbf{0 . 0 3}$ \\
\hline Shop 5 & $\mathbf{8 7 . 6 6}$ & $\mathbf{8 7 . 4 2}$ & $\mathbf{5 . 6 8}$ & $\mathbf{4 . 5 3}$ & $\mathbf{0 . 2 7}$ & $\mathbf{0 . 0 2}$ \\
\hline Shop 6 & 33.57 & 33.37 & 10.46 & 16.39 & 0.36 & 0.13 \\
\hline Shop 7 & $\mathbf{1 0 0 . 3 2}$ & $\mathbf{9 9 . 1 2}$ & $\mathbf{6 . 2 1}$ & $\mathbf{5 . 9 0}$ & $\mathbf{1 . 1 9}$ & $\mathbf{0 . 0 3}$ \\
\hline Shop 8 & 57.87 & 57.63 & 2.64 & 8.60 & 0.40 & 0.02 \\
\hline Shop 9 & 11.81 & 12.02 & 1.97 & 6.56 & 1.76 & 0.06 \\
\hline Shop 10 & $\mathbf{5 2 . 4 2}$ & $\mathbf{5 1 . 8 9}$ & $\mathbf{4 . 8 9}$ & $\mathbf{5 . 9 4}$ & $\mathbf{1 . 0 0}$ & $\mathbf{0 . 0 4}$ \\
\hline Shop 11 & $\mathbf{8 4 3 . 8 3}$ & $\mathbf{8 4 5 . 6 5}$ & $\mathbf{7 6 . 2 2}$ & $\mathbf{2 . 1 5}$ & $\mathbf{0 . 2 1}$ & $\mathbf{0 . 0 2}$ \\
\hline
\end{tabular}


Table 5: Predefined response modeling results for commercial center pilot shops.

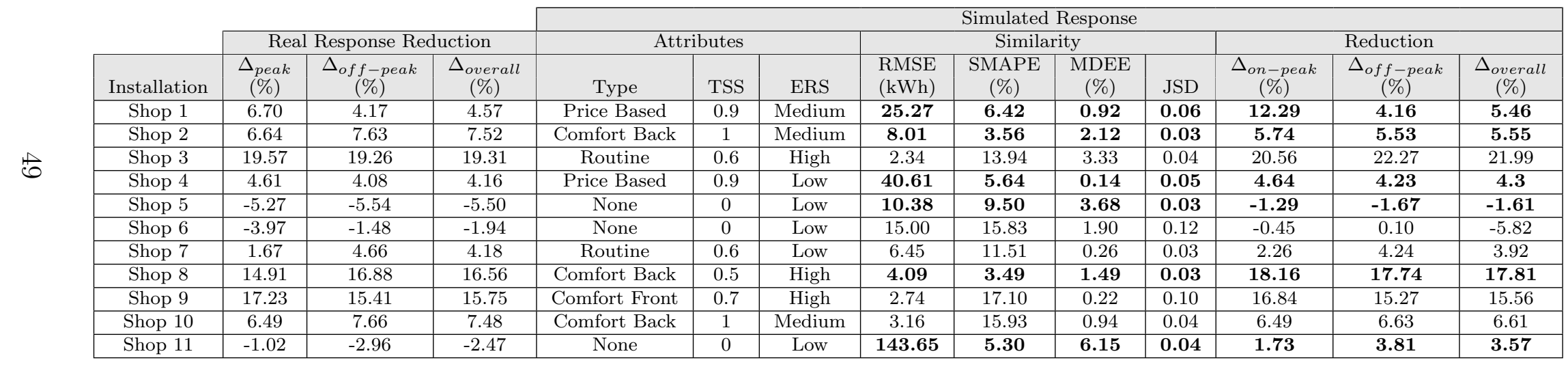

